\section{Mortalidade infantil por doenças infecciosas e parasitárias: reflexo das desigualdades sociais em um município do Nordeste do Brasil}

\author{
Infant mortality due to infectious and \\ parasitic diseases: a reflection of the social \\ inequalities in a municipality in the \\ Northeast Region of Brazil
}

Mirella Bezerra Rodrigues Vilela 1

Cristine Bonfim 2

Zulma Medeiros 3

1,3 Laboratório de Doenças Transmissíveis. Departamento de Parasitologia. Centro de Pesquisas Aggeu Magalhães. Av. Moraes Rego, s.n. Recife, PE, Brasil. CEP: 50.670-420. E-mail:mirellarod@hotmail.com

2 Fundação Joaquim Nabuco. Diretoria de Pesquisas Sociais Recife, PE, Brasil..

\begin{abstract}
Objectives: to study the relationship between the coefficient of infant mortality (CIM) due to infectious and parasitic diseases (IPD) and the social need indicator, thereby identifying geographical areas at greater risk in the municipality of Jaboatão dos Guararapes, in the State of Pernambuco, Brazil.

Methods: an ecological study was carried out, in which the units of analysis were the districts of the municipality. The mortality and live birth information systems of the Ministry of Health were used to calculate the CIM/IPD, and the social need indicator was established using socioeconomic data from the 2000 census.

Results: the CIM/IPD was 3.56 per 1000 live births and the postnatal mortality coefficient was 3.39 per 1000 live births. There was a correlation between the social need indicator and the CIM/IPD $(r=0.87$; $p=0.008 ; F=12.88$ ). It was found that there was $48 \%$ greater risk of dying from infectious and parasitic diseases before reaching one year of age $(R R=1.479)$ among the children in the stratum of greatest social need.

Conclusions: the social need indicator is a useful instrument for planning local healthcare actions and helps with the adoption measures that bring about a reduction in infant mortality. However, this should not direct attention away from the development of adequate social and economic policies for needier areas.
\end{abstract}

Key words Infant mortality, Parasitic diseases, Health inequalities, Social conditions

\section{Resumo}

Objetivos: estudar a relação entre o coeficiente de mortalidade infantil (CMI) por doenças infecciosas e parasitarias (DIP) e o indicador de carência social (ICS), identificando áreas geográficas de maior risco no município de Jaboatão dos Guararapes, Pernambuco, Brasil.

Métodos: trata-se de um estudo ecológico cujas unidades de análise foram os bairros do municipio. Os sistemas de informações de mortalidade e de nascidos vivos do Ministério da Saúde foram utilizados para o cálculo do CMI por DIP e o ICS foi construído utilizando dados socioeconômicos do Censo 2000.

Resultados: o CMI por DIP foi de 3,56 por $10^{3}$ nascidos vivos $(\mathrm{NV})$ e o coeficiente de mortalidade pósneonatal foi de 3,39 por $10^{3} \mathrm{NV}$. Houve correlação entre ICS e o CMI por DIP ( $r=0,87 ; p=0,008 ; F=12,88)$. Encontrou-se um risco $48 \%(R R=1,479)$ maior das crianças irem a óbito por DIP antes de completarem um ano no estrato de maior carência social.

Conclusões: o ICS é um útil instrumento para o planejamento de ações locais de saúde, contribuindo para a adoção de medidas que promovam a redução da mortalidade infantil, não descartando a necessidade de demandar atenção para o desenvolvimento de políticas sociais e econômicas nas áreas com maiores carências sociais.

Palavras-chave Mortalidade Infantil, Doenças parasitárias, Desigualdades em saúde, Condições sociais 


\section{Introdução}

A mortalidade infantil (MI) possui uma estreita relação com as condições socioeconômicas e de saúde da população. Por isso, o coeficiente de mortalidade infantil (CMI) é considerado um indicador clássico de saúde, pois além de mensurar o risco de morte para crianças com menos de um ano de vida, informa sobre o desenvolvimento social de uma comunidade. ${ }^{1}$ A probabilidade de sobrevivência no primeiro ano de vida associa-se com a forma de organização coletiva da sociedade, demonstrando compromisso com a sua reprodução social.1-3

No Brasil, durante as últimas décadas, foi verificado um acentuado declínio nas taxas de mortalidade infantil, sobretudo no período pós-neonatal, devido aos fatores ligados a melhorias no saneamento ambiental. ${ }^{4}$ Essa redução é um fato incontestável, todavia não se distribui de forma homogênea, ressaltando as desigualdades existentes entre os lugares e os diversos segmentos populacionais.4,5

As desigualdades em saúde não são apenas as diferenças existentes neste campo, mas consistem em um tipo particular de diferença, a qual prejudica socialmente um determinado grupo, resultando em maiores riscos para saúde desse agregado menos favorecido. 6 Em outras palavras, nem toda diferença na situação de saúde pode ser considerada iniqüidade, no entanto toda diferença ou desigualdade redutível, associada com as condições heterogêneas de vida, indubitavelmente, caracterizam iniqüidade.

Barata7 afirma que as desigualdades sociais em saúde são resultantes das diferenças produzidas pelas formas de inserção dos indivíduos nas sociedades e encontram-se relacionadas com a forma de divisão social da riqueza. Nesta medida, o processo de aferição do risco de adoecer e morrer distribuído pelos diferentes segmentos da sociedade deve ter seu arcabouço ligado às condições de vida da população. ${ }^{8}$

A relação da mortalidade infantil com as condições de vida da população já está bem estabelecida tanto na literatura internacional 5,9-12 quanto na nacional.1,13-19 Fatores de risco para essa mortalidade estão associados à pobreza e a outros elementos que incluem o inadequado abastecimento de água, as precárias condições de higiene, o saneamento deficiente, o desmame precoce e as deficiências nutricionais. ${ }^{9}$ Segundo Victora et al., ${ }^{10}$ as crianças pobres são mais expostas, com menor resistência às doenças e têm o acesso reduzido a intervenções preventivas e curativas. A análise da mortalidade infantil em nove países em desenvolvimento apontou o Brasil como o país de maior desigualdade social na mortalidade infantil.5
As doenças infecciosas e parasitárias (DIP) consistem em uma causa de óbito infantil intimamente relacionada com fatores socioeconômicos e ambientais. ${ }^{11-13}$ Para determinados grupos populacionais a sua ocorrência é mais expressiva devido às precárias condições de vida. Esse grupamento de doenças destaca-se como uma das principais causas de internações hospitalares e mortalidade infantil no Brasi1 13,14 e no mundo. ${ }^{11,12}$

Em 2005, as DIP foram responsáveis por 3707 óbitos em menores de um ano no Brasil, ocupando o terceiro lugar no ranking da MI, quando se exclui o grupo de sinais e sintomas mal definidos. ${ }^{20}$ A região Nordeste do país concentrou 1814 (48,93\%) dos óbitos em menores de um ano por DIP. Em Pernambuco, a situação é semelhante: em 2005, 321 crianças morreram por DIP, o que representou aproximadamente $10 \%$ dos óbitos nesse grupo etário no Estado.20 Quando se exclui o grupo de sinais e sintomas mal definidos, a mortalidade infantil por DIP também ocupa o terceiro lugar entre as causas de óbito em menores de um ano em Pernambuco.20

Embora Jaboatão dos Guararapes seja o segundo maior município do Estado de Pernambuco, nenhum estudo foi conduzido no sentido de identificar os diferenciais intra-urbanos que expressem o risco da mortalidade infantil por DIP, bem como sua relação com as condições sociais locais. Desta forma, este estudo justifica-se pela necessidade científica e social. O objetivo deste trabalho foi estudar a relação entre a mortalidade infantil por doenças infecciosas e parasitárias no município de Jaboatão dos Guararapes e o indicador de carência social (ICS), identificando áreas geográficas de maior risco, contribuindo para o planejamento das ações locais, em especial as medidas que promovam a redução da MI.

\section{Métodos}

A área de estudo foi constituída pelo município de Jaboatão dos Guararapes, segundo maior município do Estado de Pernambuco, localizado na Região Metropolitana do Recife, cerca de $18 \mathrm{Km}$ da capital. Atualmente tem uma população de 651.353 habitantes, sendo $97,75 \%$ urbana e distribuída numa área de $256 \mathrm{Km}^{2}$. Trata-se de um estudo ecológico, cujas unidades de análise foram compostas pelos bairros do município. A fonte de dados foi constituída pelos sistemas de informações sobre mortalidade e nascidos vivos do Ministério da Saúde no período de 2000 a 2004, coletados na Secretaria Municipal de Saúde e do Censo 2000.

Os dados referentes às condições gestacionais e 
maternas tiveram como fonte o Sistema de Informação sobre Nascidos Vivos (SINASC) do Ministério da Saúde. Para as informações sobre os óbitos foi utilizado o banco de dados do sistema de informações sobre mortalidade (SIM) do Ministério da Saúde. A variável faixa etária da mãe foi obtida através do SIM, pois esta deveria ser correspondente ao momento do óbito.

Para as informações referentes às condições demográficas e socioeconômicas, foi utilizada a base de dados do Censo 2000, disponibilizado pelo Instituto Brasileiro de Geografia e Estatística (IBGE). A partir da base de dados do SIM, realizouse um linkage entre essa e o SINASC, tomando como campos de emparelhamento as variáveis: nome da mãe, data do nascimento e sexo da criança. O objetivo deste procedimento foi garantir uma melhor qualidade dos dados. 21

Foram incluídos no estudo todos os nascidos vivos, residentes no município, que foram a óbito com menos de um ano de vida e que tiveram as DIP (Capítulo I, da 10 revisão da Classificação de Internacional Doenças - CID 10) como causa básica e/ou causas associadas do óbito, ocorridas no período entre 2000 e 2004, agrupados nas unidades de análise. A mortalidade infantil por DIP foi analisada a partir do CMI por DIP e a carência social baseada no indicador sintético de risco.

A adoção da multicausalidade (causa básica e/ou causas associadas) foi utilizada com a finalidade de captar todos os óbitos infantis que tiveram no encadeamento das causas alguma DIP, ainda que essa não tenha sido a causa básica do óbito. Este procedimento teve como referência os estudos de Puffer e Serrano 22 e Puffer, 23 que indicam esse método como indispensável para a melhoria da validade do conteúdo.

De um total de 200 óbitos por DIP em menores de um ano, 71 (35,5\%) não foram localizados no SINASC municipal. Acreditando na possibilidade de migração (no momento do nascimento a genitora era residente em outro município) buscou-se a informação no banco do SINASC da Secretaria de Saúde do Estado de Pernambuco e nesse foi possível localizar 36 dos 71 não encontrados no SINASC do município, reduzindo para 35 o quantitativo de declarações de nascidos vivos (DNV) não localizadas. A fim de recuperar dados ignorados na declaração de óbito, realizou-se uma busca ativa em domicílio e nessa foi possível resgatar informações de 16 das 28 procuradas. A partir disto, o estudo foi composto por 188 óbitos infantis por DIP, em residentes de Jaboatão dos Guararapes, no período de 2000 a 2004.
As condições sociais foram analisadas a partir do indicador de carência social (ICS), utilizando as variáveis: domicílios particulares permanentes sem abastecimento de água, domicílios particulares permanentes sem instalação sanitária, domicílios particulares permanentes sem coleta de lixo, pessoas responsáveis pelos domicílios particulares permanentes sem instrução, pessoas responsáveis pelos domicílios particulares permanentes sem rendimento nominal mensal. Esta seleção deu-se a partir de suas relações com as condições de vida e a infra-estrutura urbana, sendo capaz de representar a situação de carência social, além de permitir a identificação de áreas com maiores níveis de precariedade. 24

O ICS foi construído através da técnica de formação de escores. 24 As variáveis selecionadas foram agregadas por setor censitário (SC), computadas em valores absolutos, para em seguida se extraírem os percentuais de ocorrência (OC). Os escores (ES), como medida de ordenamento dos SC, foram elaborados a partir dessa OC. Os SC que apresentaram maiores OC em cada uma das variáveis consideradas receberam ES valor 1 e a menor OC, ES igual a 0. Os demais ES foram construídos a partir da equação:

$$
\mathrm{ES}_{\mathrm{sv}}=\left(\mathrm{OC}_{\mathrm{s}}-\mathrm{OC}_{\min , \mathrm{v}}\right) /\left(\mathrm{OC}_{\text {máx, } \mathrm{v}}-\mathrm{OC}_{\min , \mathrm{v}}\right)
$$

Onde:

$\mathrm{ES}_{\mathrm{sv}}$ representa o escore do setor censitário " $\mathrm{s} "$ em relação à variável "."; $\mathrm{OC}_{\mathrm{s}}$ representa a ocorrência da variável "v" no setor censitário "s"; $\mathrm{OC}_{\text {min, } \mathrm{v}}$ representa a ocorrência mínima para a variável "v", observada entre todos os setores censitários; $\mathrm{OC}_{\text {máx, }}$ representa a ocorrência máxima para a variável " $\mathrm{v}$ ", observada entre todos os setores censitários;

A partir desta construção, os bairros do município foram agrupados segundo áreas homogêneas de carência social, sendo os estratos 1 e 4 de menor e maior carência, respectivamente. Já os estratos 2 e 3 são de média carência social.

Com o objetivo de estabelecer a associação da mortalidade infantil por DIP e o ICS foi utilizado o coeficiente de correlação de Pearson (r), confirmado através da estatística $\mathrm{F}$, aceitando um erro $\alpha$ menor que $5 \%$.

O projeto foi aprovado pelo Comitê de Ética do Centro de Pesquisas Aggeu Magalhães (CAEE número 0042.0.095.000-06) e autorizado pela Diretoria de Epidemiologia da Secretaria de Saúde de Jaboatão dos Guararapes. 


\section{Resultados}

Dos 188 óbitos por DIP em menores de um ano, 179 ocorreram no período pós-neonatal $(95,21 \%)$ e nove no neonatal $(4,79 \%)$. O CMI por DIP foi de 3,56 por $10^{3} \mathrm{NV}$ e o CMPN (coeficiente de mortalidade pósneonatal) foi de 3,39 por $10^{3} \mathrm{NV}$. Quando se distribuiu esses óbitos por grupos homogêneos, 59 $(31,38 \%)$ situaram-se no estrato de maior carência social, $109(57,98 \%)$ no estrato de média e 20 $(10,63 \%)$ no de menor carência social.

O CMI por DIP nos estratos demonstrou que os maiores coeficientes foram encontrados nas áreas com os maiores níveis de carência social (Tabela 1). No estrato 1 (menor carência social) o CMI por DIP foi de 3,29 por $10^{3} \mathrm{NV}$, passando para 3,74 por $10^{3} \mathrm{NV}$ no estrato $2,3,86$ por $10^{3} \mathrm{NV}$ no estrato $3 \mathrm{e}$ para 4,8 por $10^{3} \mathrm{NV}$ no estrato 4 , o de maior carência social (Tabela 1).

O mapa descrito na Figura 1 apresenta os CMI por DIP ordenados por quartis de forma crescente $\mathrm{e}$ distribuídos por bairros. A Figura 2 caracteriza as diferentes condições sociais, obtendo-se a disposição geográfica dos estratos homogêneos de carência social.

Comparando-se os estratos, tem-se que o estrato de maior carência social (estrato 4) apresenta um risco $48 \%(R R=1,479)$ maior das crianças irem à óbito por DIP antes de completar um ano, em relação ao estrato de menor carência social. Este risco reduz gradativamente quando é calculado para os demais estratos, com RR de 1,17 e 1,13, respectivamente para os estratos 3 e 2 . Foi encontrada a correlação de $87 \%$ entre o ICS e o CMI por DIP nos estratos $(r=0,87 ; p=0,008 ; F=12,88)$.

Tabela 1

Distribuição do número de nascidos vivos, número de óbitos infantis, do coeficiente de mortalidade infantil por doenças infecciosas e parasitárias e do indicador de carência social nos estratos de risco. Jaboatão dos Guararapes, Pernambuco, 2000 - 2004.

\begin{tabular}{llccccc}
\hline Estratos & Risco & $\begin{array}{c}\text { Intervalos } \\
\text { interquartilicos }\end{array}$ & $\begin{array}{c}\text { Nascidos } \\
\text { vivos }\end{array}$ & $\begin{array}{c}\text { Óbitos <1 } \\
\text { ano por DIP }\end{array}$ & CMI & ICS \\
\hline 1 & Baixo & 32,461 |-40,964 & 17900 & 59 & 3,29 & 39,89 \\
2 & Médio & 40,964 |-44,632 & 16551 & 62 & 3,74 & 43,85 \\
3 & Médio & 44,632 |-52,248 & 12165 & 47 & 3,86 & 48,52 \\
4 & Alto & 52,248 |-83,239 & 4102 & 20 & 4,87 & 64,91 \\
\hline
\end{tabular}

$\mathrm{DIP}=$ doenças infecciosas e parasitárias; $\mathrm{CIM}=$ coeficiente de mortalidade infantil; ICS=indicador de carência social.
Figura 1

Distribuição do coeficiente de mortalidade infantil por doenças infecciosas e parasitárias segundo bairros. Jaboatão dos Guararapes, Pernambuco, 2000 - 2004.

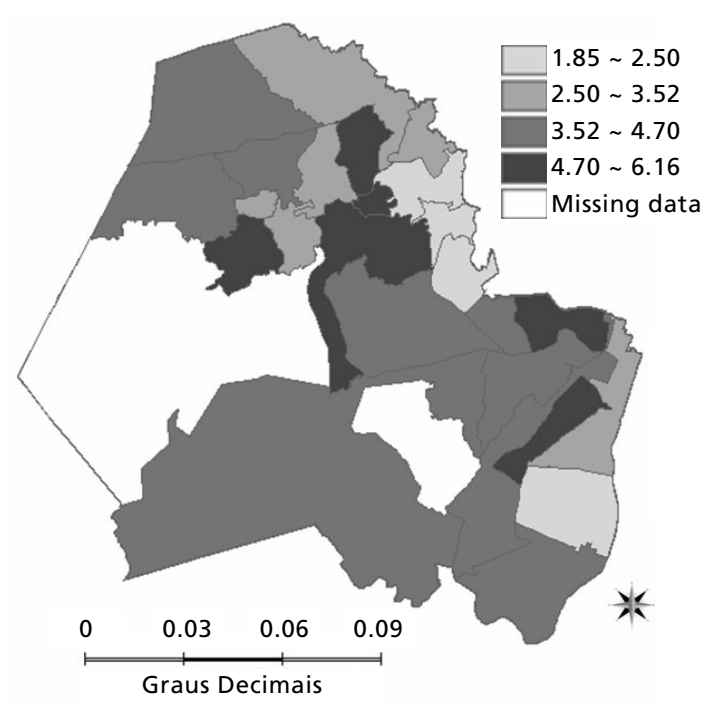

Figura 2

Distribuição do indicador de carência social segundo estratos homogêneos. Jaboatão dos Guararapes, Pernambuco, 2000.

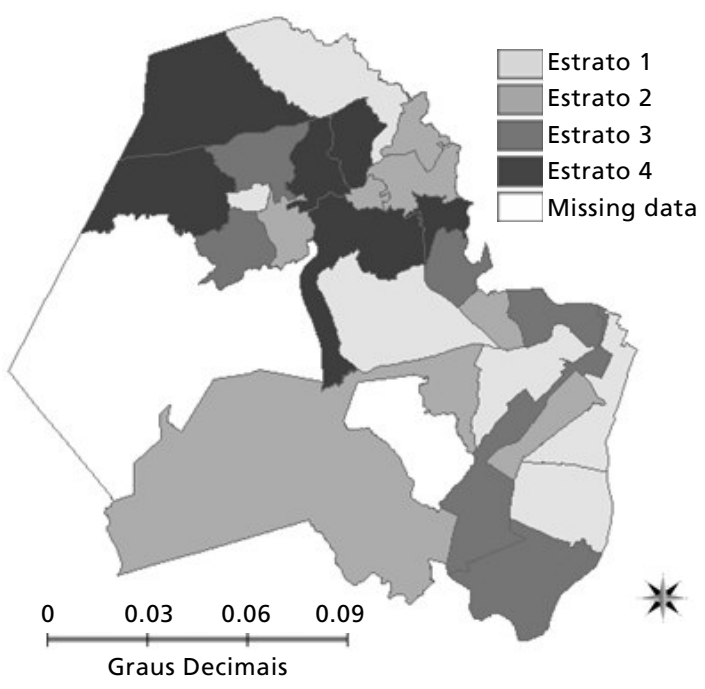




\section{Discussão}

A mortalidade infantil por DIP constitui-se um evento importante para a saúde coletiva em Jaboatão dos Guararapes. O município apresentou coeficiente acima do encontrado no Brasil $\left(1,42 / 10^{3} \mathrm{NV}\right)$ e no Nordeste $\left(2,30 / 10^{3} \mathrm{NV}\right){ }^{20}$ Segundo dados do Ministério da Saúde, entre 2000 e 2005, as DIP ocuparam o quarto lugar entre os grupamentos de causas de óbito em menores de um ano no Brasil, sendo responsável por uma média de $7,47 \%$ do universo desses óbitos. 20

Holcman et al., 2 avaliando a evolução da mortalidade infantil na Região Metropolitana de São Paulo, Estado de São Paulo, verificaram um acentuado decréscimo na taxa de mortalidade pósneonatal e uma redução exponencial da mortalidade infantil por doenças infecciosas parasitárias. O principal determinante dessa queda consistiu na ampliação da rede de abastecimento de água.

As associações entre o CMI por DIP e o ICS foram feitas com os estratos de risco, e nestes foi possível evidenciar os diferenciais intra-urbanos, retratando a relação entre o risco de morte na infância e a carência social em Jaboatão dos Guararapes. Andrade et al.,17 estudando a mortalidade infantil por cluster de condições de vida, no Estado do Paraná, verificaram que as maiores taxas de mortalidade foram encontradas nos cluster de pior condição, especialmente no componente pósneonatal. Os autores observaram um decréscimo da taxa de mortalidade à medida que o cluster se situava em um melhor nível socioeconômico.

De forma geral, vários estudos no Brasil retratam a existência de diferenciais intra-urbanos, o que permite a identificação de territórios constituídos de agregados populacionais com alto risco de MI.1,15-19 Costa et al. 15 evidenciaram que o grupamento das DIP constituiu-se na segunda principal causa de óbito infantil em todos os estratos, aumentando sua magnitude à medida que pioravam as condições de vida, encontrando o maior risco de morte infantil por essa causa no estrato de pior condição de vida. Levando em consideração que os processos envolvidos com a mortalidade inantil por DIP são complexos e dinâmicos, é necessário um contínuo acompanhamento, de forma que se disponha de informações sobre a situação de saúde no nível local.

O ICS mostrou sensibilidade na predição das áreas de risco. A principal função desse indicador consiste em possibilitar a identificação de situações de saúde desiguais entre os segmentos populacionais, orientando as ações de saúde. À medida que possibilitou discriminar grupos relativamente homogêneos da população, está oferecendo as possibilidades técnicas de monitoramento das condições de vida e saúde. Uma das vantagens desse indicador é a possibilidade de sintetizar as informações socioeconômicas, tornando-o de fácil manuseio e compreensão para os serviços de saúde.

Embora os resultados deste trabalho possam estar sujeitos a limitações metodológicas, tais como a heterogeneidade intra-agregados, a mobilidade entre os grupos e sub-notificação de óbitos infantis, com este estudo foi possível identificar as áreas do município onde residem os grupos populacionais expostos ao maior risco de ir a óbito por DIP antes de completar o primeiro ano de vida, assim como a manutenção das desigualdades, permitindo confirmar que as condições sociais detêm um papel importante na determinação dessa mortalidade. 17

Bezerra Filho et al. ${ }^{1}$ reforçam a tese de que os fatores sociais se mantêm como determinantes da mortalidade infantil, embora também destaquem a importância da adoção das ações públicas específicas à saúde da criança. Essas, por sua vez, configuram-se emergenciais e insuficientes à redução da moralidade infantil e à sua manutenção em nível mínimo possível. A continuidade dessa redução dependerá de transformações estruturais: melhor distribuição de renda, inclusão da população em programas educacionais, expansão do saneamento básico e da atenção à saúde em níveis primário e secundário. Essas medidas são exemplos de ações institucionais concretas que afetam os níveis de mortalidade infatil e redimensionam a ordem de seus determinantes. 16

Segundo Costa et al.,16 na ausência de grandes transformações sociais é válido intensificar a aplicação de ações no nível básico de saúde. Medidas como o aumento da cobertura da estratégia de saúde da família, prioridade na atenção ao pré-natal e ao parto, cuidados no período pós-neonatal fazem parte das múltiplas intervenções na atenção básica de saúde que visam à redução da mortalidade infantil. 25

Os resultados encontrados nesse trabalho evidenciaram que o ICS permite a visualização geográfica das áreas de menor e maior carência social, destacando grupos homogêneos prioritários para intervenções direcionadas, contribuindo assim para a redução das iniqüidades em saúde. Ademais, o conhecimento da distribuição da mortalidade infatil por DIP pode ser um instrumento capaz de expressar as desigualdades no município. Estudos complementares serão necessários a fim de que os métodos utilizados sejam reproduzidos no planejamento dos serviços locais de saúde.

Sugere-se adotar como estratégia o fortaleci- 
mento da vigilância epidemiológica do óbito infantil, incentivando articulações intersetoriais na discussão sobre os determinantes sociais da mortalidade por DIP, assim como a implantação do comitê municipal de prevenção da mortalidade infantil em Jaboatão dos Guararapes, que poderá ser uma relevante contribuição ao conhecimento da mortalidade infantil e conseqüentemente à adoção de medidas que visem a sua redução, não descartando a necessidade de demandar atenção para o desenvolvimento

\section{Referências}

1. Bezerra Filho JG, Kerr LFS, Miná DL, Barreto ML. Distribuição espacial da taxa de mortalidade infantil e principais determinantes no Ceará, Brasil, no período 20002002. Cad Saúde Pública. 2007; 23: 1173-85.

2. Holcman MM, Latorre MRDO, Santos JLF. Evolução da mortalidade infantil na região metropolitana de São Paulo, 1980-2000. Rev Saúde Pública. 2004; 38: 180-6.

3. Andrade CLT, Szwarcwald CL. Análise espacial da mortalidade neonatal precoce no município do Rio de Janeiro, 1995-1996. Cad Saúde Pública. 2001; 17: 1199-210.

4. Duarte CMR. Reflexos das políticas de saúde sobre as tendências da mortalidade infantil no Brasil: revisão da literatura sobre a última década. Cad Saúde Pública. 2007; 23: 1511-28.

5. Wagstaff A. Socioeconomic inequalities in child mortality: comparisons across nine developing countries. Bull World Health Org. 2000; 78: 19-29.

6. Braveman P. Health disparities and health equity: concepts and measurement. Ann Rev Public Health. 2006; 27: 16794.

7. Barata RCB. Desigualdades sociais e saúde. In: Campos GWS, Minayo MCS, Akerman M, Drumond Júnior M, Carvalho YM., organizadores. Tratado de saúde coletiva. São Paulo: HUCITEC; 2006. p. 457-86.

8. Buss PM, Pellegrini Filho A. A saúde e seus determinantes sociais. Physis (Rio J.). 2007; 17: 77-93

9. Rosenberg M. Global child health: burden of disease, achievements, and future challenges. Curr Probl Pediatr Adolesc Health Care. 2007; 37: 338-62.

10. Victora CG, Wagstaff A, Schellenberg JA, Gwatkin D, Claeson M, Habicht JP. Applying an equity lens to child health and mortality: more of the same is not enough. Lancet. 2003; 362: 233-41.

11. Cheng AC, McDonald JR, Thielman NM. Infectious diarrhea in developed and developing countries. J Clin Gastroenterol. 2005; 39: 757-73.

12. Thapar N, Sanderson IR. Diarrhoea in children: an interface between developing and developed countries. Lancet. 2004; 363: 641-53.

13. Caldeira AP, França E, Perpétuo IHO, Goulart EMA. Evolução da mortalidade infantil por causas evitáveis, Belo Horizonte, 1984-1998. Rev Saúde Pública. 2005; 39: 67-74. de políticas sociais e econômicas nas áreas com maiores carências sociais.

\section{Agradecimentos}

Agradecemos a João Quaresma por sua incansável colaboração no resgate dos dados e ao programa de pós-graduação stricto sensu do CPqAM/FIOCRUZ.

14. Guimarães ZA, Costa MCN, Paim JS, Silva LMV. Declínio e desigualdades sociais na mortalidade infantil por diarréia. Rev Soc Bras Med Trop. 2001; 34: 473-8.

15. Costa MCN, Azi PA, Paim J S, Silva LMV. Mortalidade infantil e condições de vida: a reprodução das desigualdades sociais em saúde na década de 90. Cad Saúde Pública. 2001; 17: 555-67.

16. Costa MCN, Mota ELA, Paim JS, Silva LMV, Teixeira MG, Mendes CMC. Mortalidade infantil no Brasil em períodos recentes de crise econômica. Rev Saúde Pública. 2003; 37 : 699-706.

17. Andrade SM, Soares DA, Matsuo T, Souza RKT, Mathias TAF, Iwakura MLH, Zequim MA. Condições de vida e mortalidade infantil no estado do Paraná, Brasil, 1997/2001.Cad Saúde Pública. 2006; 22: 181-9.

18. Guimarães MJB, Marques NM, Melo Filho DA, Szwarcwald CL. Condição de vida e mortalidade infantil: diferenciais intra-urbanos no Recife, Pernambuco, Brasil. Cad Saúde Pública. 2003; 19: 1413-24.

19. França E, Souza JM, Guimarães M DC, Goulart E M A, Colosimo E, Antunes CMF. Associação entre fatores socioeconômicos e mortalidade infantil por diarréia, pneumonia e desnutrição em região metropolitana do Sudeste do Brasil: um estudo caso-controle. Cad Saúde Pública. 2001; 17: 1437-47.

20. Brasil. Ministério da Saúde. DATASUS (Departamento de Informações do SUS). Informações de Saúde: mortalidade Infantil [monografia online]. [acesso em: 18 mar. 2008]. Disponível em: http://www.datasus.gov.br

21. Silva CF, Leite AJM, Almeida NMGS, Gondim RC. Fatores de risco para a mortalidade infantil em município do Nordeste do Brasil: linkage entre bancos de dados de nascidos vivos e óbitos infantis - 2000 a 2002 . Rev Bras Epidemiol. 2006; 9: 69-80.

22. Puffer RR, Serrano CV. Pattems of mortality in childhood. Washington, DC: Pan-American Health Organization; 1973. (PAHO Scientific Publications, 262).

23. Puffer RR. New approaches for epidemiologic studies of mortalitys statistics. Bull Pan-American Health Org. 1989; 23: $365-83$. 
24. Ximenes RAA, Martelli CMT, Souza WV, Lapa TM, Albuquerque MFM, Andrade ALSS, Morais Neto OL, Silva AS, Lima MLC, Portugal J L. Vigilância de doenças endêmicas em áreas urbanas: a interface entre mapas de setores censitários e indicadores de morbidade. Cad Saúde Pública. 1999; 15: 53-61.
25. Bryce J, Daelmans B, Dwivedi A, Fauveau V, Lawn JE, Mason E, Newby H, Shankar A, Starrs A, Wardlaw T. Countdown to 2015 for maternal, newborn, and child survival: the 2008 report on tracking coverage of interventions. Lancet. 2008; 371: 1247-58.

Recebido em 28 de agosto de 2007

Versão final apresentada em 20 de agosto de 2008

Aprovado em 1 setembro de 2008 